\title{
THE INVESTIGATION AND TREATMENT OF VERTIGO
}

\author{
By D. RANGeR, F.R.C.S. \\ Assistant Ear, Nose and Throat Surgeon, Middlesex Hospital
}

The investigation of a patient with vertigo should start with a careful and complete history, for in most cases this plays a very important part in making an accurate diagnosis and often it is quite impossible to interpret the findings on examination without a knowledge of the symptomatology.

Terminology on this subject varies a lot even among medical men and one can never be sure what patients mean by terms such as vertigo, giddiness and dizziness except by asking them to try and describe in simple words the exact sensations they experience. Even then it is often necessary to ask leading questions such as whether the apparent movement is rotatory or swaying or some other type.

The severity of the vertigo is no indication of the severity of the underlying lesion and in many cases the most serious cases have only minor symptoms. There does not appear to be any significant difference between the apparent rotation of objects around the patient (objective vertigo) and the apparent rotation of the patient in relation to his surroundings (subjective vertigo). Likewise there does not seem to be any practical value in discovering whether rotation is clockwise or anti-clockwise.

It should be ascertained whether the vertigo occurs in bouts with periods of complete freedom in between episodes or whether there is a more or less constant sensation of unsteadiness. In general, bouts of vertigo occurring at intervals in patients otherwise normal suggest a peripheral lesion in the labyrinth whereas constant unsteadiness is more likely to occur in central lesions.

It should be determined whether the attacks occur in any particular position. Although change of position may make any vertigo worse, certain types of vertigo occur only when the patient assumes a particular posture, such as lying in bed, and these cases of positional vertigo are discussed more fully later.

In addition the duration of the attacks should be determined. A severe bout of vertigo lasting several days which keeps the patient in bed for a week or so and keeps him off work for a few weeks is very suggestive of a sudden complete vestibular failure such as occurs, without any loss of hearing, in vestibular neuronitis. In Meniere's disease the attacks often vary enormously in severity and duration; although they may be momentary most attacks last for many minutes or for hours or even for one or two days.

Enquiry about associated symptoms such as nausea, vomiting and diarrhoea may give some indication of the severity of the vertigo and of the patient's reaction to the vertigo. Diarrhoea is a rare accompaniment and tends to occur only in the emotionally unstable. In patients who have many attacks of vertigo, associated nausea and vomiting often diminish or disappear in later attacks.

Definite loss of consciousness can occur in association with vertigo from a peripheral labyrinthine lesion, but this symptom must always raise the possibility of epilepsy. One difference which may be helpful is that a patient with a labyrinthine lesion will usually still have vertigo on regaining consciousness whereas this is seldom the case in epilepsy. In addition an epileptic will usually have lost consciousness on other occasions without having any sensation of vertigo, whereas a patient with a labyrinthine lesion will probably have had other attacks of vertigo without loss of consciousness.

As most cases of vertigo are due to a cause in the ear it is important to ask about other symptoms such as aural discharge, deafness and tinnitus. The presence of any aural discharge at the time of the vertigo immediately suggests the possibility of a mastoiditis with a peri-labyrinthitis and, possibly, an erosion of the bony labyrinth. Aural discharge at some time in the past with no discharge at the time of the vertigo is unlikely to be connected with the vertigo. Patients can be very misleading about the question of discharge, for occasionally they regard wax as discharge while others strongly deny the presence of discharge, even when it is quite obvious on inspection.

While enquiring about the presence of aural discharge, enquiry should also be made about the presence of any spots in and around the ear. Although a herpes of the vestibular ganglion is 
usually associated with a geniculate herpes and an obvious facial paralysis, some cases of herpes occur with vertigo (and deafness) and with an herpetic eruption in the ear but without any facial nerve involvement.

If there is any deafness enquiry should be made about its time of onset in relation to the vertigo and also about any fluctuation in the degree of deafness, particularly in relation to the attacks of vertigo. It is also important to ask about the presence of any distortion of hearing or any sounds which jar on the ear. In Meniere's disease the distortion of hearing may sometimes be so marked as to be the predominant feature and a history of distortion and of marked fluctuation of the deafness, whether during the attacks of vertigo or not, can be regarded as almost diagnostic of Meniere's disease.

It is important to enquire about tinnitus. Cawthorne (1953) has pointed out that although tinnitus may be a very troublesome symptom to the patient, it may be a great help to the diagnostician by giving an indication of the side of the lesion in difficult cases.

Other illnesses and also drugs may well have some bearing on the problem and the history should include details of any other illnesses or accidents. Particular attention should be paid to the question of any head injury and to the possibility of the patient having had streptomycin or large doses of quinine. It should be remembered that even quite small doses of streptomycin can cause vertigo, especially in the presence of a renal lesion. Large doses of quinine are sometimes taken in an attempt to produce a miscarriage, and a history may be difficult to obtain. In one case seen personally, permanent deafness and severe vertigo followed the taking of enormous doses of quinine for this purpose, without having any effect on the pregnancy.

Other symptoms may suggest some neurological lesion. An episode of mistiness of vision or diplopia, possibly occurring a long time before the onset of vertigo, immediately raises the possibility of multiple sclerosis while numbness in the face suggests an acoustic neuroma.

Headache may occur in patients with Meniere's disease but persistent headache must always arouse the suspicion of an intracranial neoplasm.

\section{Clinical Examination}

Ideally every patient with vertigo should have a comprehensive clinical examination which can be considered most conveniently under the headings of general, neurological and otological:

\section{General}

In most cases of vertigo a ' general ' examina- tion will not reveal any significant abnormality b obviously some findings can be of the utmost importance. The discovery of a maligna neoplasm anywhere will immediately raise tie question of cranial metastases for secondaries oftén produce a very complicated and puzzling pictu $\overrightarrow{\mathrm{E}}$ from the neurological and otological point of view. Again the findings of arteriosclerosis or hypegtension may help a lot in the diagnosis. Arf evidence to suggest chronic alcoholism or syphil will be of value and the finding of neuro-fibromata anywhere will immediately raise the possibility of an auditory neuro-fibroma. Any pallor indicative of anaemia should be noted and the patient should be assessed for the possibility that the symptonis are neurotic.

\section{Neurological}

Vertigo may occur in association with neurow logical lesions in such diverse areas as the tempora lobes, the brain stem and the cerebellum, sig obviously a complete neurological examination is called for.

Particular attention should be paid to the question of nystagmus. In purely peripher labyrinthine lesions nystagmus persists for only few weeks even after complete loss of vestibular function and the persistence of nystagmus fof longer than this must indicate a more centraly placed lesion. A vertical nystagmus indicat central leșion.

Acoustic neuromata usually have otologic symptoms and signs for some time, often yearæi before other manifestations occur and it obviously desirable to arrive at a diagnosis at that stage if possible. However, later the fifth ang seventh nerves may be involved and must bo examined carefully. Impairment of the corneat. reflex may be a sign of such involvement. Minima facial weakness may often be revealed on rapid. blinking of the eyes.

Examination of the ocular fundus must obvious form part of the examination and in many cases charting of the visual fields will be required. Romberg's test should be carried out and the gat noted.

\section{Otological}

The first essential preliminary in all otological examinations is a careful inspection of the externat auditory meatus and tympanic membranes. Aff cerumen must be removed to allow a complete view of the tympanic membranes and to prevenf any interference with subsequent tests.

Note should be made as to whether the tympanic membranes are intact or whether there is an perforation and, if so, whether the perforation io 
dry or discharging. Particular attention should be paid to any perforation in the attic region or at the posterior margin of the membrane as these are likely to be associated with a cholesteatoma with possible erosion of the lateral semicircular canal or with a temporal lobe or cerebellar abscess. If a perforation is present the tragus should be pressed sharply into the meatus with a finger so as to cause sudden compression of the air in the meatus and middle ear, and note made as to whether any nystagmus is produced. If nystagmus does occur under these circumstances the 'fistula test' is said to be positive and it implies erosion of bone surrounding the inner ear. The erosion is nearly always into the lateral semicircular canal which is most exposed to pressure from a cholesteatoma. A negative fistula sign does not necessarily exclude the possibility of a fistula.

While examining the ear careful inspection should be made of the meatus and pinna and adjacent areas of skin to see if there are any herpetic vesicles or residual scars from previous vesicles.

\section{Hearing}

It is obviously essential to examine the hearing carefully in patients with vet tigo and it is often most convenient to do this before proceeding to tests of vestibular function. Tuning fork tests give much valuable information and for routine testing it is best to use a fork of 512 cycles fitted with a base. A sounded tuning fork placed with the base on the midline of the head is heard centrally, or equally in both ears, by persons with normal hearing, but tends to be lateralized to the worse hearing ear in patients with a conductive (middle ear) deafness and to the side of the better hearing ear in patients with a perceptive (cochlear and nerve) deafness. However, in many patients, especially when the deafness has been present for a long time, no localization occurs and the tuning fork appears to be heard in the centre of the head even though there is a marked difference in hearing between the two ears.

Normally a tuning fork is heard better when the limbs of a vibrating fork are placed opposite the external auditory meatus (air conduction) than when the base is placed on the mastoid process (bone conduction). The difference is quite marked and tuning forks of most patterns can be heard by air conduction for a matter of 15 to 20 seconds after they have ceased to be heard by bone conduction. In cases with deafness which is entirely perceptive in type the same relationship is found. However, when there is some interference with the sound conducting mechanism as, for instance, in middle ear disease and in otosclerosis, then the relationship is altered and in most of these cases bone conduction is better than air conduction. However, in some slight cases of conductive deafness conduction by air and bone may be equal or there may merely be a reduction of the normal I 5 to 20 second difference between the two.

In nearly all cases in which the deafness is due to a lesion in the cochlea or in the auditory nerve, there will be found the typical signs of a perceptive deafness as outlined above. Obviously, however, these signs may be very much altered if there is also a deafness due to some other cause such as an old otitis media. In addition, confusion sometimes arises in cases with moderate or severe unilateral perceptive deafness because of the ready transmission of sound from one mastoid process across the skull to the cochlea of the opposite side when bone conduction is being tested. In this way bone conduction may appear to be better than air conduction but the true state of affairs can be demonstrated if the test is repeated with the better ear ' masked' by another sound.

Sometimes it is found that with a neurofibroma of the auditory nerve, true bone conduction is better than air conduction and Dix and Hallpike (1950) have explained this phenomenon as being due to an exudate in the inner ear interfering with the conduction of sounds through the fluids of the cochlea to the organ of Corti.

Hearing acuity may be assessed to some extent by whisper and voice tests, but a more accurate assessment by audiometry is desirable in order to detect slight degrees of deafness and to allow accurate comparison between the hearing on different occasions. Audiometry can be used for assessing the hearing of pure tones or of speech and often it is desirable to carry out both tests. It is important when testing the worse hearing ear that the better hearing ear should be adequately masked to prevent any confusion caused by the unappreciated conduction of sound to the better ear. In addition to assessing the level of hearing, a speech audiogram may have diagnostic value in deciding on the cause of the deafness. In most cases of deafness the percentage of words or syllables understood increases progressively with greater amplification, but in some cases increasing amplification beyond an optimum may actually reduce the percentage recognition and this is especially liable to occur in Meniere's disease.

In cases with a perceptive deafness it is valuable to carry out tests for loudness recruitment. This phenomenon is one in which the deafness for threshold sounds is greater than the deafness for louder sounds and, in fact, when full recruitment is present there is no deafness for loud sounds even when there is marked deafness for soft sounds. If 
one ear is normal and there is a moderate difference between the two ears at threshold the test can be carried out by a loudness balance test in which notes of different intensities are relayed alternately into each ear and note made of the intensities which seem equally loud in the two ears. In patients without any recruitment the difference between the two ears will remain the same at all intensities, whereas when recruitment is present the difference at threshold levels will be reduced as the intensities are increased. If there is any reduction of this nature there is said to be 'partial recruitment,' whereas if a stage is reached in which the same sound seems equally loud in the two ears 'full recruitment' is present. In some cases the process is carried even further and 'over recruitment' occurs.

When the loudness recruitment phenomenon was first described by Fowler (1936) it was regarded as a characteristic of a perceptive (nerve) deafness. Later, Dix, Hallpike and Hood (1948) applied the test to different types of perceptive deafness and found that full recruitment was present in 30 cases of unilateral deafness due to Meniere's disease, whereas in 20 cases of degeneration of the auditory nerve due to neurofibromata or to other tumours of the cerebello-pontine angle, recruitment was absent in 14 cases and partially present in the remaining six. In a later publication by the same authors (1949) these findings were further substantiated, but they recorded one case of an acoustic neurofibroma in which full recruitment was present. To sum up the diagnostic value of the loudness balance test it may be said that it is applicable only in cases of unilateral deafness when there is a moderate difference of hearing between the two ears, and although the presence of full recruitment is very much in favour of a diagnosis of Meniere's disease, this phenomenon can occur in other disorders and does not provide an infallible distinction between Meniere's disease and an acoustic neuroma.

On theoretical grounds it would seem possible to devise a test for the recruitment phenomenon based on one ear only and thus provide a test applicable to cases with a bilateral deafness. However, although several tests have been described none of them at present seems to be completely satisfactory.

\section{Vestibular Function}

The question of spontaneous nystagmus has already been discussed but in addition it is important to test patients for the presence of positional nystagmus, especially in patients who have momentary bouts of vertigo and if the history suggests that the vertigo is related to posture. The patient should sit on a couch with the heag turned to one side and the eyes fixed on some point such as the bridge of the observer's nose. Then wit the head supported by the examiner's hands the patient should lie down. If any nystagmus develops note should be made of its time of onse $\overrightarrow{\text {; }}$ its duration and its direction. If there is $\vec{n}$ nystagmus within a minute the patient should sif up and then repeat the manoeuvre with the heact turned to the opposite side. Finally, the patien should lie down with the head hanging straight back over the end of the couch.

Although some intermediate reactions occur? in most cases any positional nystagmus which occurs falls into one of two main types. In one type, by far the commoner, after the horizonte position has been assumed there is a latent perio of some seconds followed by vertigo and nystag mus for about 5 to 15 seconds and then no furthep nystagmus. On sitting up there may be a little vertigo and possibly. slight nystagmus in thi opposite direction. On lying down again therel may be no nystagmus or vertigo at all or else it $\mathbb{B}$ less than on the first occasion and then none on the third or fourth occasion. The phenomeno of diminishing reaction with repetition is usuals referred to as fatiguability and this type of positional vertigo is almost certainly due to a p@िrif pheral lesion in the labyrinth and tends to rum benign paroxysmal course. Some cases initiated by a head injury but most occur for $n$ apparent reason. In the second type of position vertigo, the nystagmus occurs immediately this horizontal or some intermediate position assumed and continues so long as that position maintained. On sitting up the nystagmus stop? and on lying down a second (or third or fourthetc.) time the nystagmus occurs again with un? diminished vigour. This type is nearly always associated with a lesion in the posterior foss. commonly a space occupying lesion, but it cas occur in the course of a demyelinating process.

In addition, the nystagmus induced in response to a rotating object should be observed-optokinetic or optomotor nystagmus. In genera terms peripheral labyrinthine lesions do not prob duce any interference with the normal optokinet nystagmus although in some patients sudden confs plete vestibular failure, such as occurs following labyrinthectomy, produces some impairment of optokinetic nystagmus to the same side and accentuation to the opposite side. However, apart from this, any abnormality of optokinetic nystag mus is an indication of the site of the lesion the brain stem or cerebral cortex.

Provided the tympanic membranes and externa auditory meati are intact the caloric test prof 
vides a simple and effective method of assessing vestibular function. It is best carried out by the technique described by Fitzgerald and Hallpike (1943). The lateral semicircular canal is the most readily stimulated by thermal changes and accordingly is the one chosen for routine testing. In order to place it in a vertical position, and therefore in the optimum position for the setting up of convection currents, the head should be flexed $30^{\circ}$ from the horizontal. The ears are then irrigated with water at $30^{\circ} \mathrm{C}$. for 40 seconds and afterwards at $44^{\circ} \mathrm{C}$. for the same length of time. Nystagmus is timed from the moment of starting the syringing until the nystagmus stops. If no nystagmus is elicited by this procedure the test is repeated using water at $20^{\circ} \mathrm{C}$. for a period of one minute and the nystagmus timed in the same way. If no nystagmus is produced by this stimulus it can be assumed that there can be very little, if any, remaining function.

A normal result of this caloric test is shown by responses of equal length, to within five or ten seconds, from both ears at $30^{\circ} \mathrm{C}$. and $44^{\circ} \mathrm{C}$. Provided the responses are all equal, reactions of one-and-a-half to three minutes are within normal limits but inequality in the responses is a sign of abnormality even if all the reactions lie within these periods of time. Reactions longer than three minutes are suggestive of a central cortical lesion and values of less than one-and-a-half minutes indicate impaired function. The caloric test alone cannot differentiate between impaired function due to a lesion in the labyrinth and a lesion of the vestibular nerve or nucleus.

A galvanic test of vestibular function and also rotation tests may help in the investigation of special cases but are not used as routine tests and cannot be considered in detail here. They have the advantage that they can be used whatever the state of the tympanic membranes but have the disadvantage that they are more difficult to carry out and to interpret the results obtained.

\section{Special Investigations}

A blood Wasserman reaction or Kahn test or both are obvious investigations of importance, especially in bilateral cases and particularly if there has been a rapid and progressive impairment of cochlear and vestibular function. A haemoglobin estimation should be made if anaemia is suspected and a leucocyte count and sedimentation rate in possible cases of infection.

Plain X-rays of the skull may help in the diagnosis and if there is any possibility of an acoustic neuroma, views should be taken of the internal auditory meatuses. These may sometimes be shown up best by tomography (Denny, 1955).
An electro-encephalogram may also give valuable information, especially in cases of epilepsy with a vertiginous aura.

A lumbar puncture is indicated in any central lesion of doubtful etiology and air encephalography may be necessary if there is any possibility of a space occupying lesion.

\section{Treatment}

This paper can deal only briefly with the question of treatment and for that reason it is proposed to limit the discussion to two conditions -Meniere's disease and benign paroxysmal positional vertigo. Even so it is possible to give only brief and rather dogmatic views.

\section{Meniere's Disease (Endolymphatic Hydrops)}

This is such a variable condition that it is difficult to assess accurately the value of some of the methods of treatment. It would be ideal, of course, to have a therapeutic measure which would restore the labyrinth to normal again and this is the aim of most forms of treatment. Unfortunately the wide variety of methods employed demonstrates only too clearly that there is no treatment known at present which can be relied on to achieve this and as a result it is sometimes necessary to resort to a destructive procedure which will cut off all impulses from the affected labyrinth and which can be relied on to stop the attacks of vertigo in that way.

In the present state of our knowledge it would seem that in most cases it is worth trying the effect of a restricted fluid and sodium intake and possibly also a diuretic. In addition there is some evidence that vasodilation is of value and nicotinic acid has been used for this purpose for many years. More recently other oral vasodilators have become available and they atso have their advocates. Injection of histamine, either intramuscularly or intravenously, can be employed and procaine can also be used intravenously. In view of this use of histamine it may seem surprising that there are also many advocates for the use of the antihistamine group of drugs. In addition, vasodilation can be produced by injection of the stellate ganglion or by cervical sympathectomy and this may help some patients (Lewis, 1954).

Whichever of the above treatments is adopted many of the patients are also helped by sedation with a barbiturate and a combination of phenobarbitone and theobromine often seems useful. Also, it is comforting to the patient to be reassured that the cause of his trouble lies in the ear and not in the brain.

Although the above remarks may suggest that the methods of treatment already mentioned are 
of little value it must be pointed out that most patients with Meniere's disease are able to continue living a reasonably normal life when treated on the above lines. If the attacks of vertigo do continue to interfere with the patient's life and if the condition is unilateral the attacks can be stopped by a destructive procedure as mentioned above. Impulses can be cut off from the affected labyrinth either by destruction of the labyrinth itself or by division of the vestibular portion of the auditory nerve. Destruction of the labyrinth also involves complete loss of hearing on that side and for that reason division of the vestibular portion of the nerve might seem more desirable. However, this latter operation is a much more formidable procedure with a greater morbidity and a definite mortality and in most cases there is no value to be gained in preserving the distorted remnant of hearing which remains. As a result division of the vestibular portion of the nerve is restricted to those cases which have troublesome attacks of vertigo but who still have good hearing.

\section{Benign Paroxysmal Positional Vertigo}

For this condition there is no specific cure but fortunately most cases settle down spontaneously. In the period when the condition is active the patients are helped most by appreciation of the circumstances under which an attack develops anch by realization that once an attack has occurred there is usually a period of several hours during which the offending position can be adopted with. impunity. Some patients find it possible to avoic any posture which provokes the vertigo but in the others it is often best deliberately to produce an attack at a convenient moment and then enjoy period of freedom. In this way patients with this complaint can often carry on with an occupation which involves climbing ladders, etc.

In addition, many of these patients are con- $\overrightarrow{0}$ vinced that they are helped by those antihistaminics which seem most active in motions sickness.

\section{BIBLIOGRAPHY}

CAWTHORNE, T. E. (1953), Proc. Roy Soc. Med., 46, 833. DENNY, W. R. (1955), f. Laryng., 69.

DIX, M. R., and HALLPIKE, C. S. (1950), Proc. Roy. Soc. Med., $\vec{N}$ 43,291 .

DIX, M. R., HALLPIKE, C. S., and HOOD, J. D. (1948), Ibid., DIX, M. R., HALLPIKE, C. S., and HOOD, J. D. (1949), Ibid., 42, 527 .

FITZGERALD, G., and HALLPIKE, C. S. (1942), Brain, 65, 115 FOWLER, E. P. (1936), Arch. Otolaryng., Chicago, 24, 731. LEWIS, ROLAND S. (1954), f. Laryng., 68, 636.

\section{LLOYD-LUKE}

\section{Books that enshrine profound thought}

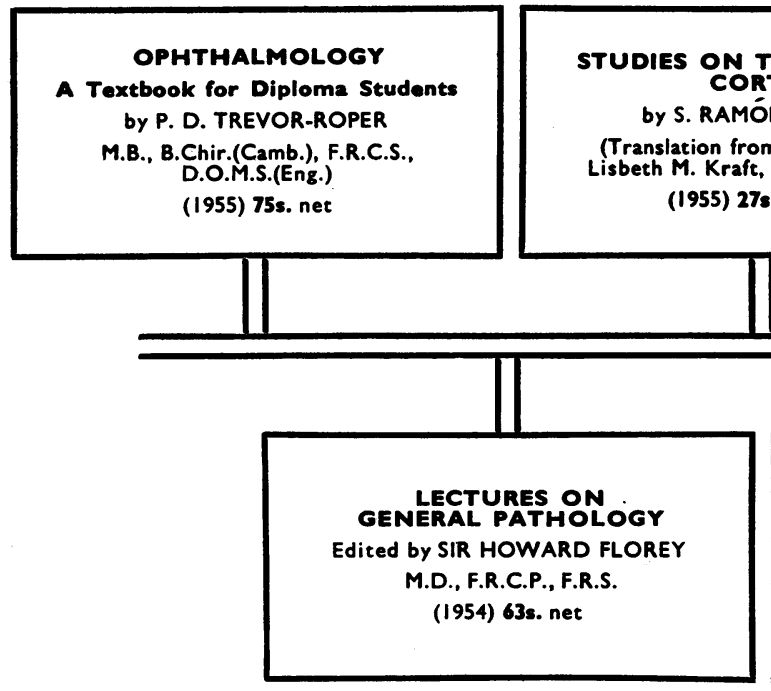

Descriptive catalogue available on request

FLUID BALANCE

IN SURGICAL PRACTICE bY L. P. LE QUESNE

D.M.(Oxon.), F.R.C.S.(Eng.)

(1954) 17s. 6d. net

\section{OBSTETRIC PROBLEMS}

bY IAN DONALD

M.B.E., M.D.(Lond.), F.R.C.O.G.

(1955) 45s. net

LLOYD-LUKE (MEDical BooKs) LTD., 49 Newman Street, W.I 